Annales Geophysicae (2003) 21: 915-922 (c) European Geosciences Union 2003

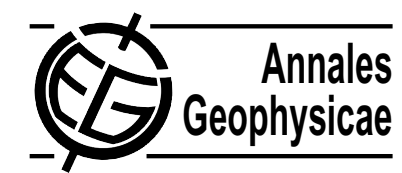

\title{
Spacecraft potential during an active experiment: a comparison of experimental results with a simple model
}

\author{
P. Žilavý, L. Přech, Z. Němeček, and J. Šafránková \\ Charles University, Faculty of Mathematics and Physics, V Holesovickach 2, 18000 Praha 8, Czech Republic
}

Received: 27 September 2001 - Revised: 22 July 2002 - Accepted: 13 September 2002

\begin{abstract}
The Active Plasma Experiment (APEX) used intensive electron beam and/or a release of the low-energy Xenon plasma to study dynamic processes in the magnetosphere and upper ionosphere. It was shown by Přech et al. (1999) that the release of the Xe plasma increases the spacecraft potential when the ambient plasma density is low, but decreases it when the density is high enough. In the present paper, a simple computer model of the dust particle charging has been adapted for a calculation of the potential difference between a planar probe and a spherical satellite moving in the ionospheric plasma. The influence of a quasineutral plasma emission on the measured spacecraft potential difference is discussed and the calculated results are compared with experimental data from the APEX project.
\end{abstract}

Key words. Ionosphere (active experiments)

\section{Introduction}

Active experiments utilizing electron beams in space have been carried out during last decade. Various problems of space plasma physics, such as generation of artificial aurora, interaction between the beam and ambient plasma, spacecraft charging, beam-plasma instability, and enhanced ionization of the surrounding gas have been investigated. One of the first experiments with plasma beam injection has been carried out on board the Porcupine rockets (Haerendel, 1976). An overdense heavy (Xenon) ion beam of conical shape was fired at a large angle with respect to the ambient magnetic field at altitudes ranging from $190 \mathrm{~km}$ to about $450 \mathrm{~km}$. Ion thruster and ambient plasma parameters in the Porcupine experiment were very similar to the conditions aboard APEX. An analysis of the experimental results given by Haeusler et al. (1986) confirms the picture of heavy ion beam propagation across the magnetized ionospheric background plasma, as developed by Haerendel and Sagdeev (1981). Specifically, the measurements confirm the presence of three successive

Correspondence to: L. Přech (lubomir.prech@mff.cuni.cz) stages of beam propagation; the diamagnetic phase, the diffusive phase, and the test particle phase. One primary observation is that the beam propagates nearly undistorted across the plasma because it is essentially depolarized. The diameter of the diamagnetic cavity created by the beam was estimated to be $\sim 3 \mathrm{~m}$, but a significant diamagnetic effect has been observed up to $15 \mathrm{~m}$. Inside this region, the beam electrons simply follow the ions into the cone of propagation. At larger distances, the motion of charged particles is controlled by the ambient magnetic field. The change in the magnetic field due to the beam current was of the order of $10 \mathrm{nT}$ outside the diamagnetic cavity and thus its influence on the particle motion is negligible.

A local enhancement of plasma density can be reached even if the neutral gas is released. Under certain conditions, the neutral gas undergoes a rapid ionization leading to the creation of a highly ionized plasma cloud. The instability (e.g. field-aligned current instabilities or current-driven electrostatic shock waves) driven by the ionization process starts, if the kinetic energy associated with the neutral gas motion perpendicular to the magnetic field exceeds the ionization potential of neutrals. This condition can be easily fulfilled if heavy atoms (usually Xe) are released from a spacecraft (Chiu, 1981). The critical ionization velocity process requires a seed charged particle population inside the neutral beam, but this population can be simply created by classical ionization processes, such as photoionization, charge exchange, thermal electron impact, etc. The tests of the proposed mechanism by neutral gas injection from rockets gave a very small ionization yield, probably due to the inefficient ion seeding of the neutral gas and/or shortness of the interaction timescale (Okuda and Choueiri, 1994). On the other hand, a ionization yield consistent with the critical ionization velocity theory has been reported by Marshall et al. (1993).

In the experiments with artificial electron beams, the spacecraft potential rises until a sufficient number of ambient electrons can be collected to balance the beam current. The spacecraft potential thus depends on the ambient plasma density (which can be artificially enhanced by emission of a 
gas) and, if a plasma density is low, it can even prevent the escape of the beam electrons, in order to maintain the current balance. Results of these experiments have shown that for low-orbiting satellites this potential does not exceed $\sim 100 \mathrm{~V}$ (for review, see, e.g. Hastings, 1995; Stone and Bonifazi, 1998).

The experiments with tethered satellites allow one to study the collection of currents by the surfaces which can be on high positive or negative potentials with respect to the ambient plasma. Gentile et al. (1998) reported the shuttle charged negatively up to $\sim 300 \mathrm{~V}$, if a long tether was connected to the shuttle. However, potentials of the order of kilovolts have been reported in high altitudes (Managadze et al., 1988) where the density of the ambient plasma is low. An injection of the neutral gas or low-temperature plasma has been regarded as one of the most promising ways to neutralize vehicle charging. This injection is able to maintain the potential in the range of tens of volts (Němeček et al., 1997) in the electron beam experiment.

The influence of the injection of the neutral/ionized Xenon from the spacecraft on its potential was investigated by Přech et al. (1999). It was found that the release of the neutral Xe does not change the spacecraft potential at ionospheric heights (500-3000 km), but the change in the spacecraft potential induced by the release of the Xe plasma depends linearly on the logarithm of the ambient plasma density. In this case, the change in the spacecraft potential was positive for low plasma densities and negative when the density of ambient plasma was high. The observed changes were qualitatively attributed to the current of electrons leaving the region occupied by the released Xe plasma.

We have developed a simple computer model of the spacecraft charging under conditions of the active experiment and compared the results of this model with experimental data from Přech et al. (1999). The basic procedures of the model were originally developed for computation of the charging of dust particles immersed into plasma (Žilavý et al., 1997), but this problem is similar to the charging of the spacecraft, if different scales are taken into account.

A motivation behind this work was to explain why the release of the Xe plasma cloud from the satellite changes its potential in both directions, as it was found in the APEX experiment.

\section{Experimental arrangement of the APEX experiment}

The APEX project was launched on 18 December 1991, and moved along the nearly polar orbit with apogee $3080 \mathrm{~km}$, perigee $450 \mathrm{~km}$, and inclination $82.5^{\circ}$. The spacecraft was three-axis stabilized with the longitudinal axis always perpendicular to the Earth's surface. The spacecraft carried an electron gun which provided the controlled injection of an emitted beam of $8 \mathrm{keV}$ and a peak current of $100 \mathrm{~mA}$, and a xenon plasma generator which injected either neutral Xe or low-energy Xe plasma. The Xe release rate was about $3 \mathrm{mg} / \mathrm{s}$. To produce an ion beam, the neutral Xe was released

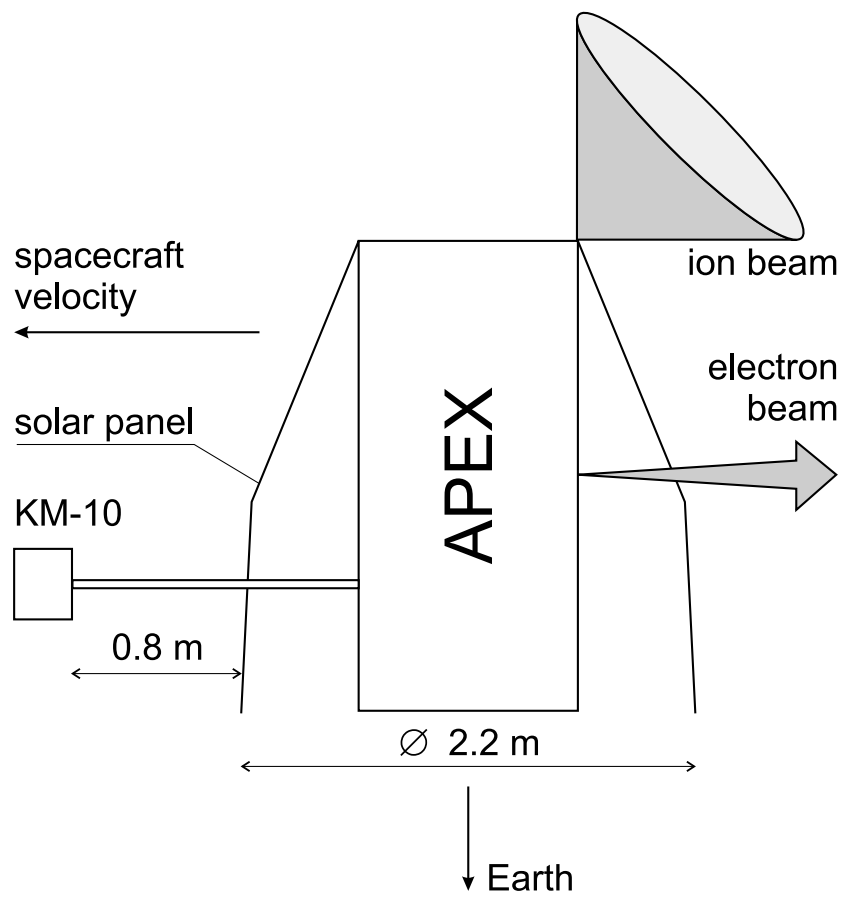

Fig. 1. Schematic drawing of the APEX payload configuration (with locations of the electron gun, plasma generator, solar panels, and $\mathrm{KM}-10)$.

through an ionizer (ion thruster) which produced about $10^{19}$ ions/s into a $\sim 90^{\circ}$ cone. The axis of the cone was inclined at $22.5^{\circ}$ to the orbital plane and directed $45^{\circ}$ upward from the Earth. The initial ion velocity corresponds to a bulk kinetic energy of $\sim 200 \mathrm{eV}$ and the total ion current can reach $2 \mathrm{~A}$. The ion beam itself was neutralized by means of an auxiliary hollow cathode discharge as the electron source. The injector worked in several different modes. All modes started with the neutral Xe; after some delay (usually $\sim 50 \mathrm{~s}$ ) the ionizer was switched on for $99 \mathrm{~s}$. This sequence was repeated until the whole system was switched off. The ionizer could be modulated by a square wave modulation with different frequencies (details in Němeček et al., 1997; Dokukin, 1992).

Ionospheric plasma parameters were measured by the KM-10 device (Afonin et al., 1994) which used four nickel planar probes with diameters of $\sim 55 \mathrm{~mm}$ surrounded by guard rings $(58 / 71 \mathrm{~mm})$, and three ion traps. A conducting box $(175 \times 175 \times 95 \mathrm{~mm})$ with sensors was located $\sim 0.8 \mathrm{~m}$ in front of the nearest solar panel in the spacecraft velocity direction. It was isolated from the holder (aluminiumalloy boom) and its potential was kept on the floating potential by an amplifier. This potential was measured by one of the probes oriented in the ram direction. Since the ram ion current was in the range $10^{-9}-10^{-5} \mathrm{~A}$ and the input current of the amplifier was less than $10^{-12} \mathrm{~A}$, the probe can be considered as floating. The corresponding circuit was designated for continuous measurement of the potential difference between the probe and spacecraft, up to $\pm 90 \mathrm{~V}$.

The other three planar probes of the KM-10 device were 
dedicated to the measurement of the three perpendicular components of the electron temperature by the RF method. The ion traps (RPA and driftmeter) had an entrance grid on the floating potential and thus, no disturbing electric field acted on the plasma in the vicinity of KM-10. A schematic drawing of the satellite configuration with locations of the electron gun, plasma generator, solar panels, and KM-10 probes are presented in Fig. 1.

Fast measurements of energy and angular parameters of charged particles were realized by the PEAS spectrometer. It consisted of two identical sensor units, AP-1 and AP-2, which cover two perpendicular planes in the energy range from $50 \mathrm{eV}$ to $30 \mathrm{keV}$. This range was divided into 64 (or 16) steps in the logarithmic scale and the time resolution of charged particle measurements was $2.6 \mathrm{~s}$ (Němeček et al., 1997).

\section{Introduction on the charging of isolated bodies in the ionosphere}

Parameters of the ionosphere plasma are highly variable. They vary with local times, latitudes, geomagnetic activity, solar cycle, etc., but the most important parameter is the altitude. The density of ionospheric plasma decreases with a distance from the Earth, being approximately $10^{12} \mathrm{~m}^{-3}$ at $500 \mathrm{~km}$ and $10^{8} \mathrm{~m}^{-3}$ at $3000 \mathrm{~km}$ of altitude. The electron and ion temperatures are of the order or less than $1 \mathrm{eV}$. The ion composition depends also on a distance from the Earth. In the range from 200 up to $1000 \mathrm{~km}, \mathrm{O}^{+}$dominates, above $1000 \mathrm{~km}$, the main components are $\mathrm{H}^{+}$and $\mathrm{He}^{+}$(e.g. Richmond, 1982).

For the interpretation of the results of active experiments with charged particle injections, it is important to determine the change in the spacecraft potential given by the charge balance of the incoming and outgoing particles. A body immersed into an ionospheric plasma is electrically charged due to a collection of electron $\left(I_{e}\right)$ and ion $\left(I_{i}\right)$ currents from the surrounding plasma, photoemission $\left(I_{p h}\right)$ or secondary emission induced by the electrons $\left(I_{\text {see }}\right)$ or ions $\left(I_{\text {sei }}\right)$. All these currents depend on the body potential, $\Phi$. The equilibrium surface potential of the body is given by the requirement:

$I(\Phi)=I_{e}(\Phi)+I_{i}(\Phi)+I_{p h}(\Phi)+I_{\text {see }}(\Phi)+I_{\text {sei }}(\Phi)=0$.

We suppose an isolated sphere (with radius, $R$ ) moving with velocity $v \sim 7 \mathrm{~km} . \mathrm{s}^{-1}$ in a quiet ionospheric plasma (not in auroral regions). Neglecting the photoemission current, $I_{p h}$, as well as other emission currents (secondary electron emission current induced by electrons and ions $I_{s e c}$, $\left.I_{\text {sei }}, \ldots\right)$ because the energy of ambient plasma is low, and following Whipple (1981) and Allen (1992), we can (using a thin sheath approximation) estimate the expression for the electron collection current:

$I_{e}(\Phi)=4 \pi R^{2} \cdot(-e) \cdot n \cdot \sqrt{\frac{k T_{e}}{2 \pi m_{e}}} \cdot \begin{cases}\left(1+\frac{e \Phi}{k T_{e}}\right) ; & \Phi>0 \\ \mathrm{e}^{\frac{e \Phi}{k_{e}}} ; & \Phi<0,\end{cases}$ where $e$ is the elementary charge, $m_{e}$ is mass of the electron, and $k$ is Boltzmann constant. $T_{e}$ and $n$ are the electron temperature and density, respectively. We assume the quasineutral plasma and thus, $n_{i}=n_{e}=n$.

The electron current is not affected by the drift velocity between the sphere and plasma because $v \ll c_{e}$, where $c_{e}$ is the thermal speed of electrons. The electrons impact the sphere from all directions. On the other hand, when $v \gg c_{i}$ ( $c_{i}$ is the thermal speed of plasma ions), we can write for the plasma ion current:

$$
I_{i}(\Phi)= \begin{cases}\pi R^{2} \cdot q \cdot n \cdot v \cdot\left(1-\frac{q \Phi}{\frac{1}{2} m_{i} v^{2}}\right) & \left(q \Phi<\frac{1}{2} m_{i} v^{2}\right) \\ 0 ; & \left(q \Phi \geq \frac{1}{2} m_{i} v^{2}\right),\end{cases}
$$

where $q$ and $m_{i}$ are the charge and the mass of plasma ions. From the satellite point of view, the ions are assumed as a parallel beam of particles (see also Fig. 3). If we suppose that:

$q \Phi \ll \frac{1}{2} m_{i} v^{2}$,

then we can derive from Eqs. (1), (2) and (3):

$\Phi=\frac{k T_{e}}{e} \cdot \ln \left(v \cdot \frac{A_{i}}{A_{e}} \cdot \sqrt{\frac{2 \pi m_{e}}{k T_{e}}}\right)$

which is the formula (e.g. Anderson et al., 1994) for the estimation of an equilibrium potential of a satellite moving in the Earth's ionosphere. $A_{i} / A_{e}$ is the ratio of effective ion and electron collection areas. This ratio is equal to $1 / 4$ for a spherical body. In the case of hydrogen plasma and satellite potentials above $10 \mathrm{~V}$, relation (4) is not fulfilled and thus, we have to consider complete Eq. (3). It should be noted that the aforementioned estimation neglects a photocurrent. The problem of the photocurrent is rather complex. It is deeply connected with the spacecraft construction because different parts of the spacecraft surface have different emission properties and the angle between the surface and direction to the Sun changes along an orbit. For this reason, we will deal with intervals when the satellite was in the Earth's shadow only.

As it follows from the above equations, the electron temperature is the very important parameter for the equilibrium potential setting. However, all currents depend on the density and, as it follows from global ionospheric models (Daniell et al., 1995), the density cannot be considered as an independent quantity. We could use the relation between the temperature and density from the model, but we have used an alternative way: we have chosen the APEX experiment data taken from middle latitudes and from these we determined the relationship between the density and temperature. The experimental data are shown as dots in Fig. 2. The data were measured by the KM-10 Langmuir probe experiment on 24 January 1992 between 12:30 and 13:30 UT (APEX orbit No. 454). Measurements started at $480 \mathrm{~km}$ and finished 


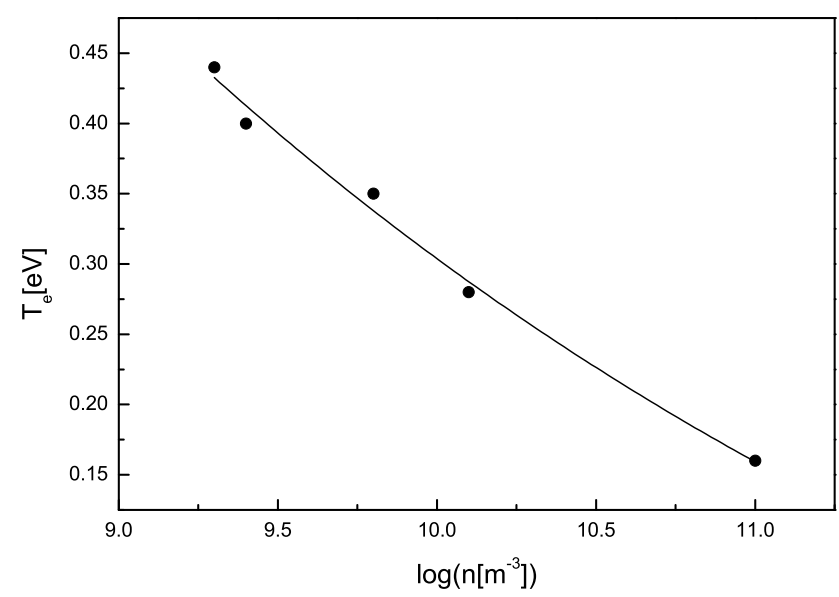

Fig. 2. The electron temperature of quiet ionospheric plasma as a function of its density.

at $3100 \mathrm{~km}$ of the altitude. The data were fitted by the function (6) which is used in our further calculations:

$T_{e}=-0.27+0.50 \cdot \exp \left(-\frac{\log (n)-10.47}{3.48}\right)$.

We think that this approach reflects, in a degree, the actual conditions during the time period under study.

\section{A simple model of an active experiment}

We suppose that the investigated system consists of a planar probe (PR), a satellite (SAT), and a Xe quasi-neutral plasma cloud (PC) released from the satellite. For the sake of simplicity, we will consider the satellite as well as plasma cloud as conducting spheres which are on the same potential ("connected by a wire"), as it is seen in Fig. 3 which presents a schematic drawing of our current system. We assume that any element of our current system does not influence the other.

For electron and ion $\left(I_{P R e}, I_{P R i}\right.$, respectively) currents on the circular planar probe, we can (under assumption $\Phi_{P R}<$ $0)$ write equations:

$$
\begin{aligned}
& I_{P R e}=\pi r^{2} \cdot(-e) \cdot n \cdot \sqrt{\frac{k T_{e}}{2 \pi m_{e}}} \cdot \mathrm{e}^{\frac{e \Phi_{P R}}{k T_{e}}} \\
& I_{P R i}=\pi r^{2} \cdot q \cdot n \cdot v \cdot\left(1-\frac{q \Phi_{P R}}{\frac{1}{2} m_{i} v^{2}}\right),
\end{aligned}
$$

where $r$ is the probe radius. The total current on the probe is then given as:

$$
I_{P R}\left(\Phi_{P R}\right)=I_{P R e}\left(\Phi_{P R}\right)+I_{P R i}\left(\Phi_{P R}\right)
$$

As we note above, we assume that the satellite and the plasma cloud are conductively connected. We assume
$\Phi_{\mathrm{SAT}}=\Phi_{P C}=\Phi$, and thus, the total current to the system is:

$$
\begin{aligned}
I(\Phi) & =I_{S A T e}^{(A)}(\Phi)+I_{S A T i}^{(A)}(\Phi)+I_{P C e}^{(A)}(\Phi)+I_{P C i}^{(A)}(\Phi) \\
& +I_{P C e}^{(B)}(\Phi)+I_{P C i}^{(B)}(\Phi)
\end{aligned}
$$

In this equation, the superscript (A) refers to the currents falling on the satellite or plasma cloud from the ambient plasma. The electron currents $\left(I_{S A T e}^{(A)}, I_{P C e}^{(A)}\right)$ are computed according to Eq. (2), the ion currents $\left(I_{S A T i}^{(A)}, I_{P C i}^{(A)}\right)$ according to Eq. (3).

The superscript (B) denotes currents outgoing from the Xe plasma cloud. If we assume the plasma with the Maxwellian distribution, the electron component can be determined from Žilavý et al. (1997):

$$
I_{P C e}^{(B)}(\Phi)=\left\{\begin{array}{cc}
I_{0} \cdot\left[\frac{2}{\sqrt{\pi}} \cdot \sqrt{\frac{e \Phi}{2 k T_{P C e}}} \cdot \mathrm{e}^{-\frac{e \Phi}{2 k T P C e}}\right. & \\
\left.-\operatorname{erf}\left(\sqrt{\frac{e \Phi}{2 k T_{P C e}}}\right)+1\right] ; & (\Phi>0) \\
I_{0} ; & (\Phi \leq 0),
\end{array}\right.
$$

where $I_{0}$ is the thermal current of electrons leaving the plasma cloud at its zero potential. The similar expression can be written for the Xe ion current, $I_{P C i}^{(B)}$. We suppose that the plasma cloud is isothermic and thus, $T_{P C e}=T_{P C i}=T_{P C}$.

For a final calculation of the equilibrium potential corresponding to a given ambient plasma density and temperature, we have used a standard method in which we integrate numerically the total current on and from the body, i.e. Eqs. (8) and (9):

$Q=\int_{0}^{t_{1}} I(\Phi(t)) d t$

and we test the fulfillment of the requirement:

$\left.\frac{d Q}{d t}\right|_{t_{1}}<\varepsilon$.

This approach requires knowledge of the relation between the charge on the body $Q$ and its potential $\Phi$. We can write $Q=\Phi \cdot C$ in vacuum, but the capacitance $C$ of a body is a function of its potential in the plasma. However, we are searching for an equilibrium state and Eqs. (11) and (12) lead to the right value of the potential, provided that $C$ is a monotonous function of $\Phi$.

\section{A comparison of model results with experimental data}

As a first step, we consider the current system consisting of the probe and satellite, i.e. without the Xe plasma release, to test the ability of our model. Under this assumption, the results do not depend on the probe and sphere radii. 


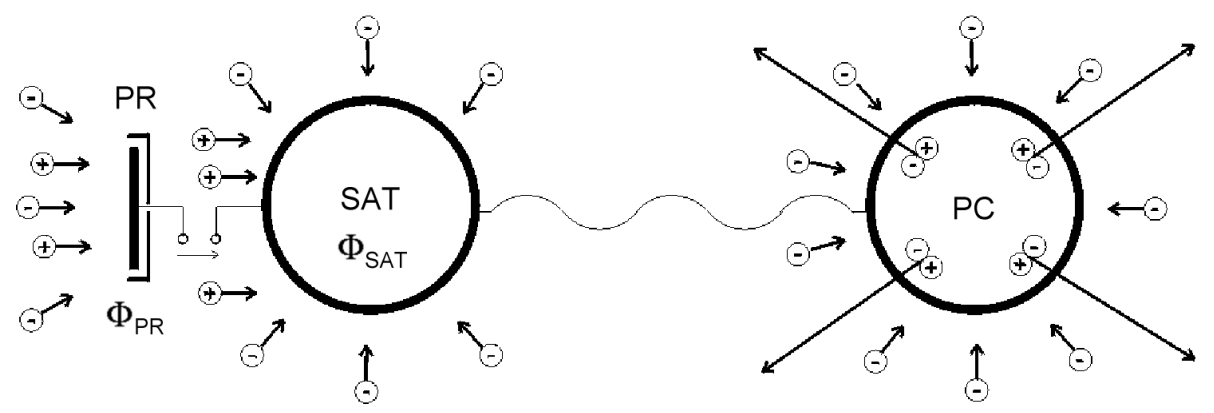

Fig. 3. Schematic drawing of our current system.

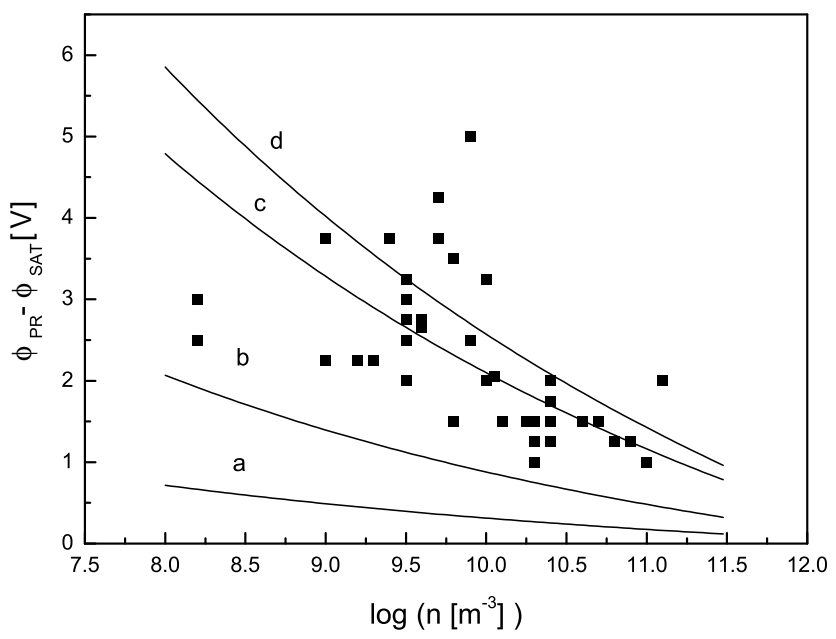

Fig. 4. Potential difference between the probe and the satellite as a function of ambient plasma density.

Since the only measurable quantity is the potential difference between the probe and satellite, we plotted these differences as a function of the plasma density in Fig. 4, curve $a$. We can see that calculated values are significantly lower than experimental data taken from Přech et al. (1999) which are plotted as dots in the figure. However, the equations, that we are using were derived in the thin sheath approximation. This approximation does not influence the computed ion current substantially, but the effective collection area for electrons is larger in real experimental conditions due to the fact that they are collected by the whole surface of the sheath. For example, a thin 22-m long boom used for the satellite stabilization does not enhance the ion collection area because it contributes only by $\sim 2 \%$ to the total cross section. The Debye length, which is a measure of the sheath thickness, can be as high as $0.2 \mathrm{~m}$ in apogee of the satellite, and the effective collection area of this boom for electrons can exceed the collection area of the satellite itself.

In order to show that even this effect is negligible, we have increased the collection area for electrons 10 times and computed the potential differences again. The result is plotted as the curve $b$ in Fig. 4. From a comparison of both $a$ and $b$ curves, we can see that the effect is relatively small and thus, we will use the spherical approximation in further calculations.

The experimental values of the temperature (Fig. 2) were estimated from the measurements at low energies under the assumption that the electron distribution function is Maxwellian. These values are consistent with ionospheric models (e.g. Daniell et al., 1995) and describe the behaviour of the principal electron component. However, the ionospheric plasma contains high-energy electrons which enhance the tail of a distribution function. This increases the effective temperature of the plasma. We can show it using data from the PEAS spectrometer (AP-2 sensor) obtained at the altitude $2600 \mathrm{~km}$ (corresponding plasma density $n \sim 10^{8} \mathrm{~m}^{-3}$ ). Figure 5 shows the counts measured on four lowest energies during passive intervals, as well as during the release of the Xe plasma. These intervals are denoted as Xe+ in Fig. 5. We will show that the ratio of these counts can be used for the determination of the temperature of the tail of the energy distribution.

In the case of the Maxwellian distribution function, the density of electrons with energy in a small range, $\Delta E$ is:

$\Delta n(E)=\frac{2 \cdot n}{\sqrt{\pi} k T_{e}} \cdot \sqrt{\frac{E}{k T_{e}}} \cdot \mathrm{e}^{-\frac{E}{k T_{e}}} \cdot \Delta E$.

Because $\Delta E$ is proportional to $E$ for electrostatic analyzers, the counts of the PEAS spectrometer can be written as:

$\Delta I(E)=C \cdot \Delta n(E) \cdot E^{2}$.

The constant $C$ is given by the geometrical factor of the analyzer. For the ratio of the currents $I_{1}$ and $I_{2}$ corresponding to two energies $E_{1}$ and $E_{2}$, we obtain:

$\frac{I_{1}}{I_{2}}=\frac{E_{1}^{5 / 2}}{E_{2}^{5 / 2}} \cdot \exp \left(\frac{E_{2}-E_{1}}{k T_{e}}\right)$

and consequently:

$$
k T_{e}=\frac{E_{2}-E_{1}}{\ln \left(\frac{I_{1}}{I_{2}} \cdot \frac{E_{2}^{5 / 2}}{E_{1}^{5 / 2}}\right) .}
$$




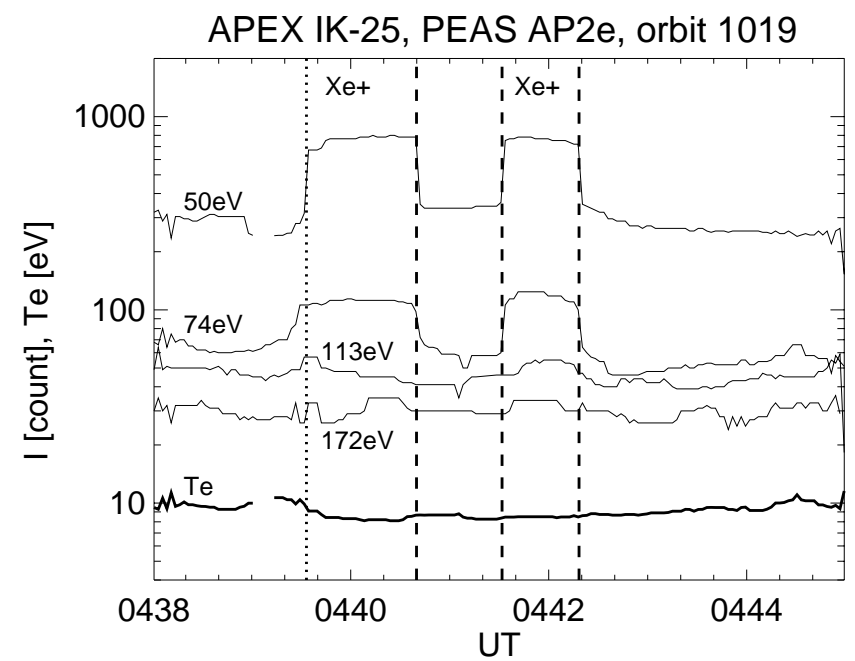

Fig. 5. Electron currents at four different energies measured by the PEAS spectrometer during a sequence of the Xe plasma release. The heavy line shows the electron temperature computed from Eq. (16) using counts on two lowest energies. The intervals of the Xe plasma release are denoted as $\mathrm{Xe}^{+}$.

For two energies, $E_{1}=74 \mathrm{eV}$ and $E_{2}=50 \mathrm{eV}$ and counts according to Fig. 5, we calculated $k T_{e}=8.5 \mathrm{eV}$ during the "passive" time interval from 04:38 to 04:39 UT. The temperature does not vary substantially with the time and thus, the release of the Xe plasma does not influence the temperature of the ambient plasma. This result will be used later for the calculations of the spacecraft potential during active experiments. We would like to note that the energies shown in Fig. 5 are given with respect to the spacecraft potential. For computation of the electron temperature according to Eq. (16), the correction on the spacecraft potential should be taken into account. The enhanced counts during the active intervals are a consequence of the change in the spacecraft potential, which will be discussed later.

The above estimation shows that the distribution function of the ionospheric plasma is not Maxwellian and that it is necessary to consider its high-energy tail which is responsible for the satellite charging. We suggest that the effect of the high-energy tail can be described as an effective rise in the plasma temperature. However, the measurements in the $\mathrm{eV}$ energy range provide a temperature less than $0.5 \mathrm{eV}$ (see Fig. 2) and the temperature determined in the range 50$70 \mathrm{eV}$ is about twenty times higher. As a number of electrons in the range of tens of $\mathrm{eV}$ is small and the majority of lowenergy electrons cannot overcome the satellite potential, the temperature determining the satellite potential would be estimated in the energy range $10-50 \mathrm{eV}$, which is not covered by the measurements. However, the spacecraft floating potential is a function of the temperature (see Eq. 5) and thus, we can use it for the determination of an "effective temperature". We multiplied the temperature in Eq. (6) by a factor $R$ and tried to find such values of $R$ which provide a best

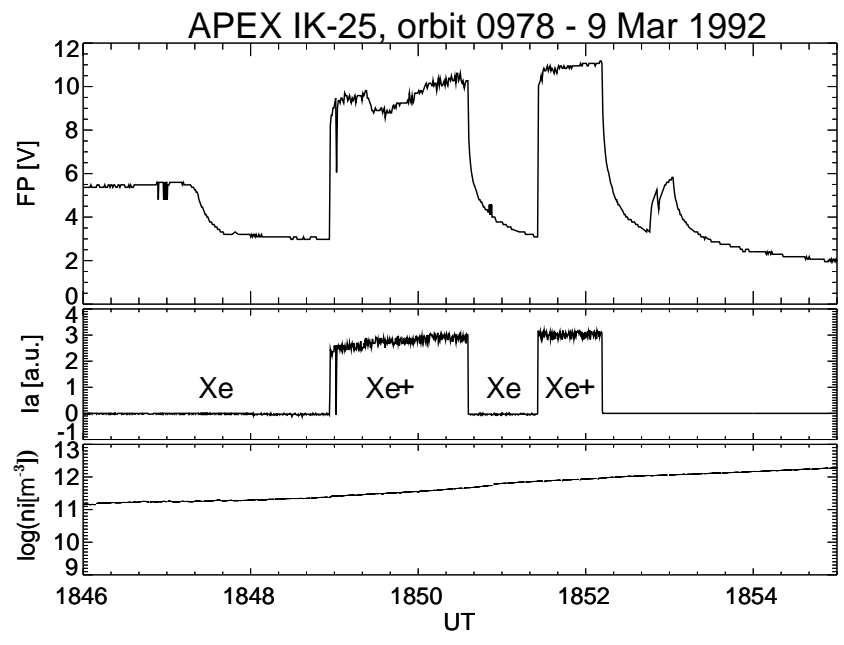

Fig. 6. Floating probe potential (vs s/c) during a sequence of neutral and ionized Xe plasma releases. From top to bottom: $F P$ - floating probe potential, I $a$ - mean Xe plasma current, log $n i$ - ambient plasma density.

fit of the experimental data in Fig. 4. One can see that the curve $c(R=8)$ represents such a fit. In order to show that the procedure is not too sensitive to the value of $R$, we also plotted the curve $d$ (for $R=10$ ) in the figure. This procedure provides an estimation of the effective temperature of the halo part of electron distribution which is responsible for a spacecraft charging. The temperature determined this way is close (about one half) to the temperature determined from the electron spectrometer for higher energies and thus, we can hope that the change in the spacecraft potential in a range of several volts, which will be investigated later, cannot be influenced significantly by a temperature determination.

As a second step, we will consider the whole current system, i.e. the probe, satellite, and the plasma cloud. Figure 6 , where the floating probe potential with the density of an ambient plasma and information on the Xe plasma injector mode are plotted, presents the changes in the spacecraft potential during the release of the neutral/ionized Xe.

The initial potential is $5.5 \mathrm{~V}$. The first change at 18:47:20 UT occurs when the spacecraft enters the Earth's shadow and floating probe potential (FP) falls to $\sim 3 \mathrm{~V}$. The release of neutral Xe was switched on at 18:48 UT, but it does not change the potential. At 18:49 UT, the plasma injector starts to release the $\mathrm{Xe}^{+}$ions and the probe potential (with respect to the spacecraft) rises to $\sim 10 \mathrm{~V}$. When the injector is switched off (18:50:35 UT), the potential relaxes to a background value. These changes repeat during the second injection period. The gradually increasing value of the potential during the plasma injection is correlated with the increasing density of the ambient plasma shown in the bottom panel.

However, the background level of FP gradually decreases with the ambient plasma density. It is about $\sim 4 \mathrm{~V}$ for the den- 


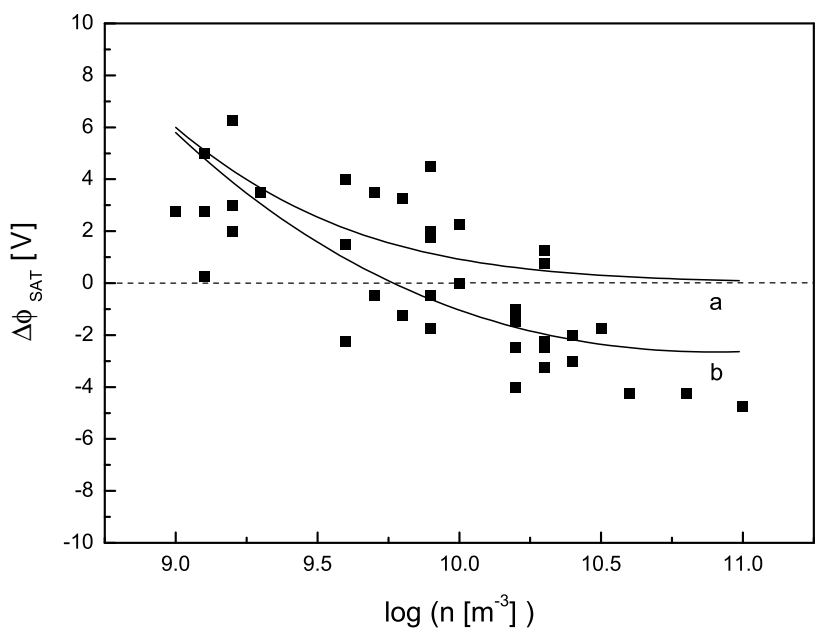

Fig. 7. The change in the satellite potential caused by a quasineutral plasma emission as a function of the ambient plasma density.

sity $\sim 1.5 \times 10^{11} \mathrm{~m}^{-3}$ (approx. at 18:48:00 UT) and $\sim 2 \mathrm{~V}$ for density $\sim 2 \times 10^{12} \mathrm{~m}^{-3}$ (at 18:54:00 UT). This trend is in accordance with our calculations shown in Fig. 4. From this it follows that the difference between satellite potentials in passive and active regimes is a function of the ambient plasma density. This difference, $\Delta \Phi$, was extensively studied by Přech et al. (1999). The data were taken from 12 January 1992 to 15 March 1992 at altitudes from 800 to $2600 \mathrm{~km}$, and it was shown that $\Delta \Phi$ decreases nearly exponentially (linearly in a semilog scale) with increasing ambient plasma density. $\Delta \Phi$ further increases with a frequency of the modulation of the injector. The experimental data from the aforementioned paper are plotted in Fig. 7 as dots, together with two curves calculated according to our model. The line $a$ was calculated under the following boundary conditions: the satellite radius, $R=1 \mathrm{~m}$, the Xe plasma cloud radius, $R_{P C}=10 \mathrm{~m}$, temperature and density on the border of the Xe plasma cloud, $T_{\mathrm{Xe}}=0.2 \mathrm{eV}, n_{\mathrm{Xe}}=3 \times 10^{9} \mathrm{~m}^{-3}$. We have chosen the diameter of the Xe plasma cloud independent of the ambient plasma density because we assume that if the density on the boundary of the expanding plasma cloud will be lower than that used in the calculations, such cloud cannot be treated as a conductor connected to the spacecraft.

In calculations, we have solved Eq. (11) under requirement (12) and considered all currents in Eq. (9). For the electron temperature of the ambient plasma, expression (6) multiplied by a factor 8 was used. As we can see, the calculations can explain a high positive value of $\Delta \Phi_{\mathrm{SAT}}$ observed for low plasma densities. The $\Delta \Phi_{\mathrm{SAT}}$ changes are caused by electrons leaving the plasma cloud. Their influence is substantial when the density of the ambient plasma is low and asymptotically approaches zero for high plasma densities, because the number of these electrons is independent on the density of the ambient plasma. The contribution of the Xe ions to the overall current balance is negligible and thus, the present form of the model cannot explain the observed negative val- ues of $\Delta \Phi_{\mathrm{SAT}}$.

To achieve the negative values, we turned the ambient plasma ion current on the Xe plasma cloud down to zero. In the experiment, a partial decreasing of the ion current can occur, for example, due to the "wake" behind the satellite. However, the main reason why the ambient ions cannot contribute significantly to the overall current balance is the energetics. To show it, we divide the whole range of altitudes into three sub-ranges. At high altitudes, where the ambient plasma density is low, a majority of ions are light $\mathrm{H}^{+}$ ions. The potential of the cloud can be slightly positive (compare Figs. 4 and 7) and thus, these ions can be captured by a local potential minimum inside the cloud, but their contribution to the current balance is negligible. At middle altitudes, the potential of the cloud is near zero and mass composition changes in favour of heavier $\mathrm{O}^{+}$ions. These ions carry $\sim 4 \mathrm{eV}$ of ram energy with respect to the spacecraft and thus, penetrate through the cloud. The potential of the cloud becomes negative at low altitudes and thus, the ions penetrate through it without any contribution to the current balance. Moreover, all ions are accelerated by a local electric field inside the cloud. Such ions thus move faster and their density decreases in order to maintain the current balance. This effect would further increase the negative potential of the cloud. However, the influence of the ion kinetics on the charge balance inside the cloud is out of the scope of the present paper.

The calculated result for zero ion current on the Xe plasma cloud is in Fig. 7, line $b$. We would like to note that the changing composition of the ionospheric plasma with altitudes (i.e. roughly with density) would modify the effect of the capture of ambient ions. At high altitudes (low densities), a majority of ions are light $\mathrm{H}^{+}$ions which have not enough energy to leave the cloud, whereas $\mathrm{O}^{+}$ions are a prevailing component at lower altitudes. The changing mass composition would cause the real dependence to lie somewhere in between curves $a$ and $b$.

As the current of ions from the ambient plasma is small when the density is low, and $\mathrm{O}^{+}$dominates at high densities, one can expect major corrections at the middle of the density interval and thus, the real dependence will be close to the straight line used by Přech et al. (1999) for a fit of experimental data.

We would like to note that our results cannot be applied to magnetospheric experiments (e.g. Comfort et al., 1998) where other currents representing the photocurrent and secondary electron current are of principal importance.

\section{Conclusion}

The potential difference between a planar probe and spherical satellite has been calculated as a function of the ambient plasma density. Calculated results have been compared with the real experimental data (Přech et al., 1999). It has been shown that the potential of a body immersed in the ionospheric plasma is determined by the temperature of a tail of 
the electron-energy distribution, whereas use of the temperature of a body of distribution leads to values of the potential significantly lower than those observed.

We have found that such a simple computer model that does not consider the magnetic field can explain positive, as well as negative changes in the satellite potential due to the quasi-neutral Xe plasma emission. The change in the spacecraft potential induced by the release of the Xe plasma is in order of volts. We suggest that the released plasma creates a dense conducting cloud behind the spacecraft, which can effectively collect electrons from the ambient plasma. The dimension of this cloud is probably comparable with the dimensions of the "diamagnetic phase" of the plasma cloud expansion proposed by Haeusler et al. (1986).

The ionospheric $\mathrm{O}^{+}$ions cannot be collected by the cloud because their energy is too high and thus, the contribution of the ion current to the charge balance is small. The computed potential difference thus depends mainly on the currents of electrons leaving the Xe plasma cloud and electrons collected from the ambient plasma and can be either negative (if the number of electrons collected from the ambient plasma is higher than the number leaving the cloud) or positive. The collected electron current rises linearly with the ambient plasma density, but this rising current causes an increase in the negative spacecraft potential. The result is the observed relationship between $\log n$ and $\Delta \Phi$ (Fig. 7).

The comparison of model results with experimental data has shown an importance of the non-Maxwellian distribution of ionospheric electrons for the estimation of the spacecraft potential, as well as the influence of the mass composition of the ambient plasma on the potential of the satellite releasing a plasma cloud.

Acknowledgement. The present work was supported by the Czech Grant Agency under Contract 202/99/M004, by the Grant Agency of Charles University under Contract 176/01, and partly by the Research Project, MSM 113200004.

Topical Editor G. Chanteur thanks J. Raitt and another referee for their help in evaluating this paper.

\section{References}

Afonin, V. V., Grechnev, K. V., Ershova V. A., Roste O. Z., Smirnova, N. F., Shultchishin, J. A., and Smilauer, J.: Ion composition and temperature of the ionosphere in the maximum of 22-cycle solar activity from Intercosmos-24 satellite (Active project),(in Russian), Cosmic Research, 32, 82-94, 1994.

Allen, J. E.: Probe theory - the orbital motion approach, Physica Scripta, 45, 497-503, 1992.

Anderson, P. C., Hanson, W. B., Coley, W. R., and Hoegy, W. R.: Spacecraft potential effects on the Dynamics Explorer 2 satellite, J. Geophys. Res., 99, 3985-3997, 1994.

Comfort, R. H., Moore, T. E., Craven, P.D., Pollock, C. J., Mozer, F. S., and Williamson, W. S.: Spacecraft potential control by the plasma source instrument on the POLAR satellite, J. Spacecraft and Rockets, 35, 845-849, 1998.

Chiu, Y. T.: Theoretical aspects of heavy ion injections in the magnetosphere: A review, Adv. Space. Res., 1, 291-304, 1981.
Daniell, R. E., Brown, L. D., Anderson, D. N., Fox M. W., Doherty, P. H., Decker, D. T., Sojka, J. J., and Schunk, R. W.: Parametrized ionospheric model - a global ionospheric parametrization based on first principles models, Radio Sci., 30 , 1499-1510, 1995.

Dokukin, V. S.: The APEX project scientific facility orbital complex, in Project APEX. Scientific purposes, simulation, technology and equipment of experiment, Nauka, Moscow, 16-28, 1992.

Gentile, L. C., Burke, W. J., Huang, C. Y., Machuzak, J. S., Hardy, D. A., Olson, D. G., Gilchrist, B. E., Lebreton, J.-P., and Bonifazi, C.: Negative shuttle charging during TSS-1R, Geophys. Res. Lett., 25, 433-436, 1998.

Haerendel, G. (Ed): Project Porcupine, Summary of scientific objectives and instrumentation, report, Max-Planck-Inst. für Phys. und Astrophys., Ins. für extraterr. Phys., Garching, 1976.

Haerendel, G. and Sagdeev, R. Z.: Artificial plasma jet in the ionosphere, Adv. Space Res., 1, 29-35, 1981.

Hastings, D. E.: A review of plasma interactions with spacecraft in low Earth orbit, J. Geophys. Res., 100, 14 457-14 483, 1995.

Haeusler, B., Treumann, R. A., Bauer, O. H., Haerendel, G., Bush, R., Carlson, C. W., Theile, B., Kelley, M. C., Dokukin, V. S., and Ruzhin, Y. Y.: Observations of the artificially injected Porcupine xenon ion beam in the ionosphere, J. Geophys. Res., 91, 287303, 1986.

Managadze, G. G., Balebanov, V. M., Burchudladze, A. A., Gagua, T. I., Leonov, N. A., Lyakhov, S. B., Martinson, A. A., Mayorov, A. D., Riedler, W. K., Friedrich, M. F., Torkar, K. M., Laliashvili, A. N., Klos, Z., and Zbyszynsky, Z.: Potential observations of an electron-emitting rocket payload and other related plasma measurements, Planet. Space Sci., 32, 399-410, 1988.

Marshall, J. A., Burch, J. L., Choueiri, E. Y., and Kawashima, N.: CIV experiments on ATLAS-1, Geophys. Res. Lett., 20, 499502, 1993.

Myers, N. B., Raitt, W. J., White, A. B., Banks, P. M., Gilchrist, B. E., and Sasaki, S.: Vehicle charging effects during electron beam emission from the CHARGE-2 experiment, J. Spacecraft and Rockets, 27, 25-37, 1990.

Němeček Z., Šafránková, J., Přech, L., Šimůnek, J., Šmilauer, J., Gringauz, K. I., Shutte, N. M., Teltsov, M. V., Marjin, B. V., Ruzhin, Y. Y., and Dokukin, V. S.: Artificial electron and ion beam effects: APEX experiment, J. Geophys. Res., 102, 22012212, 1997.

Okuda, H. and Choueiri, E. Y.: Numerical simulation of neutral gas release experiments in the ionosphere, Phys. Plasmas, 1, 16691675, 1994.

Přech, L., Němeček, Z., Šafránková, J., Šimůnek, J., and Šmilauer, J.: Artifical ion beam effects on spacecraft potential, Adv. Space Res., 44, 1027-1032, 1999.

Richmond, A. D.: Thermosphere dynamics and electrodynamic, in: Solar-Terrestrial Physics (Eds) Carovillano, R. L. and Forbes, J. M., Reidel, Dordrecht, Boston, and Lancaster, 1982.

Stone, N. H. and Bonifazi, C.: The TSS-1R mission: Overview and scientific context, Geophys. Res. Lett., 25, 409-412, 1998.

Whipple, E. C.: Potentials of surfaces in space, Rev. Prog. Phys., 44, 1197-1250, 1981.

Žilavý, P., Sternovský, Z., Němeček, Z., and Šafránková, J.: Study of the charging of dust particles: Computer simulation, in: WDS'97, Proceedings of Contribution Papers: Physics of plasmas and ionized media, (Ed. Šafránková, J.), MFF UK, Prague, 262-267, 1997. 